\title{
Using of Glycated Albumin Rather than Glycated Hemoglobin for assessing Glycaemic Control in Hemodialysis Patients with Type II Diabetes Mellitus
}

\author{
Salahaldin M. Fahad ${ }^{1}$, Rashied M. Rashied ${ }^{2}$, Waleed N. Jaffal ${ }^{3}$ \\ ${ }^{1}$ PH.D. Student, Department of Biology, College of Sciences, University of Anbar, Iraq, ${ }^{2}$ Assistant Professor \\ (Animal Physiology), Department of Biotechnology, College of Sciences, University of Anbar. Iraq, ${ }^{3}$ Assistant \\ Professor (Consultant Urologist), Department of Surgery, College of Medicine, University of Anbar, Iraq
}

\begin{abstract}
Diabetes mellitus (DM) is a global epidemic that is correlated with long-term damage and causes dysfunction of many organs like the kidney leading to diabetic nephropathy (DN) which need for heamodialysis (HD) to protect progressive renal decline. This study was designed to evaluate the significance of glycated albumin (GA), compared with fasting blood sugar (FBS) and glycated hemoglobin (HbA1c), were evaluated as an indicator of glycemic control, also determinant of insulin. This study was conducted on 95 patients at age rang (30-70) years, and patients include (35) HD patients with diabetes, (35) HD patients without diabetes and (25) Type 2 diabetes mellitus, who attended atRamadi General Hospital and (25) samples as control included in this study. This study showed that $\mathrm{HbA} 1 \mathrm{c} \%$ was significantly lower than simultaneous FBS and GA $\%$ in HD patients in comparison with in diabetic patients and control, as illustrated in significantly shallow slope of regression line between FBS with HbA1c and FBS with GA. Also, GA\% was nearly corresponding between before and after one hemodialysis in HD patients compared with HbAlc.In conclusion, GA provides a better measure to estimate glycemic control in HD patients with diabetes compared with HbAlc.
\end{abstract}

Keywords: Chronic kidney disease, Diabetes mellitus, Glycated Albumin, HbA1C, Hemodialysis.

\section{Introduction}

Diabetes mellitus (DM) is a metabolic disorder of carbohydrate leading persistent high level of blood glucose due to insufficient amount of insulin. Chronic hyperglycemia is associated with damage and causes dysfunction of several organs such as kidney ${ }^{(1 ; 2)}$. Chronic kidney disease (CKD) is progressive loss of kidney function over a period of months or years ${ }^{(2)}$. The single most common of CKD is diabetic nephropathy (DN), which occurs as a result of microangiopathy caused by diabetes and is one of the most important disorders leading to renal failure. Strict glycemic control

\footnotetext{
Corresponding Author:

Salahaldin M. Fahad

PH.D. Student, Department of Biology, College of

Sciences, University of Anbar, Iraq

e-mail: salahalden615@gmail.com
}

is beneficial in preventing complications of diabetic nephropathy ${ }^{(3)}$. Heamodialysis (HD) is successful in prolonging the life of end-stage renal disease (ESRD) patients. Selecting reliable clinical biomarkers to monitor glycemic control is critical in HD patients with diabetes. Glycated hemoglobin (HbAlc) and glycated albumin (GA) are biomarkers used for estimating glycemic control $^{(4)}$. At present, HbAlc is widely used as a gold standard index for glycemic control in clinical practice. However, the HbA1c levels may be erroneous in patients with CKD because of numerous factors as anemia and the management of erythropoietin (EPO). GA has been developed as an index for glycemic control and it measures specifically the glycation product of albumin. To date, GA has been suggested as a more reliable and sensitive glycemic index to replace $\mathrm{HbA} 1 \mathrm{c}$ in diabetic patients with $\mathrm{CKD}^{(5)}$, because it is not influenced by anemia and associated treatments ${ }^{(4)}$. The main aim of this study was assess whether GA might provide a 
better indicator than $\mathrm{HbA} 1 \mathrm{c}$ for glycemic control in $\mathrm{HD}$ patients with diabetes.

\section{Materials and Method}

The Specimens collection were started from April, 2019 till end of June 2019. HD patient with diabetes (35) and HD patient without diabetes (35) samples were collected from the Industrial Renal department and T2DM patients (25)from the Diabetes Center for Treatment at Ramadi General Hospital. For the purpose of comparison, (25) samples as control. Before the collection of samples, a careful history was taken from each patient according to a questionnaire. From each patient and control, $(5 \mathrm{ml})$ of blood were obtained by venipuncture. The blood samples were divided into two aliquots; $2 \mathrm{ml}$ was dispensed in EDTA tube and used for $\mathrm{HbA1c}$ which determination by fast ion-exchange resin separation method for the detection of $\mathrm{HbAlc}$ in human blood according to ${ }^{(6),}$ which provided from human company/Germany. While $3 \mathrm{ml}$ was dispensed in plain tube to collect serum, the serum were used in estimation FBS and GA. FBS determination according to ${ }^{(7)}$ method, which provided with kit from linear company/Spain. Determination of GAby ELISA: According to Cat.No:YHB1374method as cited by manufacturing company kit, which provided from Shanghai company/China. This kit uses enzyme-linked immune sorbent assay based on biotin double antibody sandwich technology to assay Human GA. To measure the percent of GA, the conversion formula supplied with kit was applied to all subjects; the formula is GA\%= $([\mathrm{GA}$ concentration/Total albumin]/1.14 $\times 100)+2.9$.

Statistical: The statistical analyses were carried out using SPSS version 25. One way ANOVA test was used to find means and standard deviation (SD) for all variables of the study. The difference significances in proportions analyzed by LSD test. The correlations between variables were confirmed by Pearson correlation analysis. P-value $>0.05$ were considered to be significant.

\section{Results and Discussion}

Variation of FBS Levels during Study Period of 60 day: The results in table (1) corresponds with Inaba et al., ${ }^{(8)}$ who recorded that FBS in HD patients with diabetes for three measures was stale. These results no corresponds with Lin et al., ${ }^{(9)}$ who found that the mean FBS levels after the first year of hemodialysis were stable.

Table (1): The mean levels of FBS between the four study groups.

\begin{tabular}{|l|c|c|c|c|c|}
\hline Groups & N & $\begin{array}{c}\text { Mean } \pm \text { SD FBS1 (60 } \\
\text { day) Before of GA \& } \\
\text { HbA1C meas. }\end{array}$ & $\begin{array}{c}\text { Mean } \pm \text { SD FBS 2 (30 } \\
\text { day) Before of GA \& } \\
\text { HbA1C meas. }\end{array}$ & $\begin{array}{c}\text { Mean } \pm \text { SD FBS 3 (0 } \\
\text { day) at GA \& HbA1C } \\
\text { meas. }\end{array}$ & $\begin{array}{c}\text { T- test P } \\
\text { value }\end{array}$ \\
\hline Control & 25 & $89 \pm 19$ & $88.4 \pm 18.6$ & $88.2 \pm 16.3$ & $0.165^{\mathrm{NS}}$ \\
\hline Diabetes & 25 & $218.5 \pm 44.6$ & $216.8 \pm 46.3$ & $211.8 \pm 48.4$ & $0.547^{\mathrm{NS}}$ \\
\hline HD patients with diabetes & 35 & $189.8 \pm 58.8$ & $188.5 \pm 61.5$ & $187.6 \pm 60.8$ & $0.371^{\mathrm{NS}}$ \\
\hline HD patients without diabetes & 35 & $94.8 \pm 11.6$ & $92 \pm 12.4$ & $90.7 \pm 9.6$ & $0.252^{\mathrm{NS}}$ \\
\hline Total & $\mathbf{1 2 0}$ & $\mathbf{1 4 8 . 3} \pm 70.7$ & $\mathbf{1 4 6 . 2} \pm \mathbf{6 8 . 5}$ & $\mathbf{1 4 4 . 5} \pm \mathbf{6 8 . 7}$ & $\mathbf{0 . 1 2 4}$ \\
\hline
\end{tabular}

*NS: Mean non-significant differences at $\mathrm{P} \leq 0.05$.

These results suggested that glycemic control of control group and patients groups were stable during the study period (60 days) before determined of GA and $\mathrm{HbAlc}$ and a single determination immediately before HD can be indicative of glycemic control in HD diabetes patients. The stable glycemic control during the preceding 2 mo may negate the different impact of acute glycemic control changes between $\mathrm{HbAlc}$ and GA in this study. In the first month of hemodialysis, the average FBS levels reflect the initial degree of glycemic control achieved at this stage of hemodialysis therapy. The 3- months FBS levels reflect the long-term glycemic control of hemodialysis therapy and the general condition of the hemodialysis patient at this stage ${ }^{(9)}$.

Determination of Average Fasting Blood Sugar (FBS) Level: Table (2) showed average of FBS was a significant increase in diabetic group $(215 \pm 11.1)$ 
$\mathrm{mg} / \mathrm{dl}$, followed by HD patients with diabetes group $(188.7 \pm 18.39) \mathrm{mg} / \mathrm{dl}$ compared with other groups, while the average of FBS was decreased in control group and HD patients without diabetes were $(88.5 \pm 4.88$ and $92.2 \pm 2.7) \mathrm{mg} / \mathrm{dl}$ respectively compared with other groups.

Table (2): The mean of average FBS between four study groups.

\begin{tabular}{|l|c|c|}
\hline Groups & N & Mean \pm SD of Average FBSNV(70-100) mg/dl \\
\hline Control & 25 & $88.5 \pm 4.88^{\mathrm{a}}$ \\
\hline Diabetes & 25 & $215 \pm 11.1^{\mathrm{b}}$ \\
\hline HD patients with diabetes & 35 & $188.7 \pm 18.39^{\mathrm{c}}$ \\
\hline HD patients without diabetes & 35 & $92.2 \pm 2.7^{\mathrm{a}}$ \\
\hline Total & $\mathbf{1 2 0}$ & $\mathbf{1 4 6 . 1} \pm \mathbf{2 6 . 3 6}$ \\
\hline
\end{tabular}

*Different Letters $(\mathrm{a}, \mathrm{b}, \mathrm{c})$ : Means significant difference at $\mathrm{P} \leq 0.05$.

The results in table (2) agreement with Khalaf, (10); Abid Hammed, ${ }^{(11)}$ who recorded that the significantly higher in diabetic patients and HD patients with diabetic compared to control, but our results disagreed in another part of study by Abid Hammed, ${ }^{(11)}$ when demonstrated non-significant difference between diabetic patients and HD patients with diabetes. The level of blood glucose normally represents balance between the inflow of glucose into blood and its uptake by the tissue. This result demonstrated an increase in gluconeogenesis in diabetic patients which is the predominant mechanism responsible for increased hepatic glucose output in patients with T2DM and it is correlated with fasting plasma glucose level. The fasting hyperglycemia has been result from an increased rate of glucose production which including increased rate of hepatic glycogenolysis and gluconeogenesis. While in HD patients, FBS affects by dialysis fluid, and changes in glucose levels in the dialysis fluid during and after hemodialysis ${ }^{(10 ; 11)}$.

Determination of Glycoheamoglobin (HbA1): Table (3) showed mean HbA1C was a significantly increased in diabetic patients $(10.41 \pm 1.16) \%$ compared with other groups, also a significant increase in HD patients with diabetes $(8.194 \pm 1.22) \%$. While the mean of $\mathrm{HbA1C}$ was decreased in HD patients without diabetes and control were $(5 \pm 1.23$ and $5.31 \pm 0.76) \%$ respectively.

Table (3): The mean of HbA1c percent between four study groups.

\begin{tabular}{|l|c|c|}
\hline Groups & N & Mean \pm SD of HbA1C NV(4.5-6.2)\% \\
\hline Control & 25 & $5.31 \pm 0.76^{\mathrm{a}}$ \\
\hline Diabetes & 25 & $10.41 \pm 1.16^{\mathrm{b}}$ \\
\hline HD patients with diabetes & 35 & $8.194 \pm 1.22^{\mathrm{c}}$ \\
\hline HD patients without diabetes & 35 & $5 \pm 1.23^{\mathrm{d}}$ \\
\hline Total & $\mathbf{1 2 0}$ & $\mathbf{7 . 1 3} \pm \mathbf{1 . 8 6}$ \\
\hline
\end{tabular}

*Different letters $(\mathrm{a}, \mathrm{b}, \mathrm{c})$ : Means significant difference at $\mathrm{P} \leq 0.05$.

The results in table (3) agreed with Hadi and Sultan, ${ }^{(12)}$ who recorded that the HbA1C increased in diabetic patients compared with control. Also agreed with Abid Hammed, ${ }^{(11)}$ who showed that $\mathrm{HbA1C}$ was significantly higher among T2DM (10.6 \pm 1.6$) \%$ and
HD patients with diabetes $(8.5 \pm 1.8) \%$, compared with control $(4.8 \pm 0.7) \%$. The rise in the HbA1c levels was associated with the increasing level of FBS in diabetic groups, indicating that testing of HbAlc appears as a measure of chronic hyperglycemia in diabetic patients. 
The HbA1c has been used as an objective marker of average glycemic control because the levels of HbAlc in the blood reflect the glucose levels which erythrocyte has been exposed during its lifetime of the $\mathrm{RBC}^{(13)}$.Thus, the elevation of HbA1C levels in our study indicates reduced control of blood glucose levels ${ }^{(10)}$.
Determination of GA: Table (4) showed that the mean GA\% was a significantly increased in diabetic patients and HD patients with diabetes were $(30.5 \pm 4.15$ and $29.2 \pm 4.5) \%$ respectively, while the mean of GA\% was decreased in control $(16.1 \pm 1.8) \%$ followed by HD patients without diabetes $(17 \pm 1.12) \%$.

Table (4): The mean of GA percent between four study groups.

\begin{tabular}{|l|c|c|}
\hline Groups & N & Mean \pm SD of GA\%, NV $(>\mathbf{1 8}) \mathbf{\%}$ \\
\hline Control & 25 & $16.1 \pm 1.8^{\mathrm{a}}$ \\
\hline Diabetes & 25 & $30.5 \pm 4.15^{\mathrm{b}}$ \\
\hline HD patients with diabetes & 35 & $29.2 \pm 4.5^{\mathrm{b}}$ \\
\hline HD patients without diabetes & 35 & $17 \pm 1.12^{\mathrm{a}}$ \\
\hline Total & $\mathbf{1 2 0}$ & $\mathbf{2 3 . 7 8} \pm \mathbf{1 3 . 0 5}$ \\
\hline
\end{tabular}

*Different Letters $(\mathrm{a}, \mathrm{b}, \mathrm{c})$ : Means significant difference at $\mathrm{P} \leq 0.05$.

Results in table (4) consistent with Lu et al., ${ }^{(3)}$; Chen et al., (14) who recorded that mean of GA\% increase in HD patients with diabetes compared with HD patients without diabetic and control. And consistent with Fukami et al., ${ }^{(15)}$ who recorded that the mean of GA\% was increased in diabetes patients compared with control. GA concentrations could accurately reflect the glycaemic control in such patients without being influenced by anaemia and hypoalbuminemia. GA may be desirable in diabetic patients with ESRD complicated with both renal anaemia and hypoalbuminemia. While in HD patients without diabetes, increased GA and impaired renal function should be closely monitored for the development of arterial stiffness ${ }^{(15)}$.

\section{Correlation between FBS with GA\% or FBS} with $\mathrm{HbA1c \%}$ in study groups.

There were significant and positive correlations between FBS with GA\% ( $\mathrm{r}=0.34, \mathrm{P}<0.01$; Figure (1) $\mathrm{A} 1)$ and $(\mathrm{r}=0.371, \mathrm{P}<0.01$; Figure (2) A2) or FBS with $\mathrm{HbA} 1 \mathrm{c} \%$ ( $\mathrm{r}=0.459, \mathrm{P}<0.01$; Figure (1) B1) and ( $\mathrm{r}$ $=0.356, \mathrm{P}<0.01$; Figure (2) B2) in diabetic patients and HD patients with diabetic respectively. As shown, the correlation between FBS and GA\% was similar between HD patients with diabetes and diabetic patients, but FBS and $\mathrm{HbA} 1 \mathrm{c} \%$ seemed to be significantly lower in HD patients with diabetes than in diabetic patients.

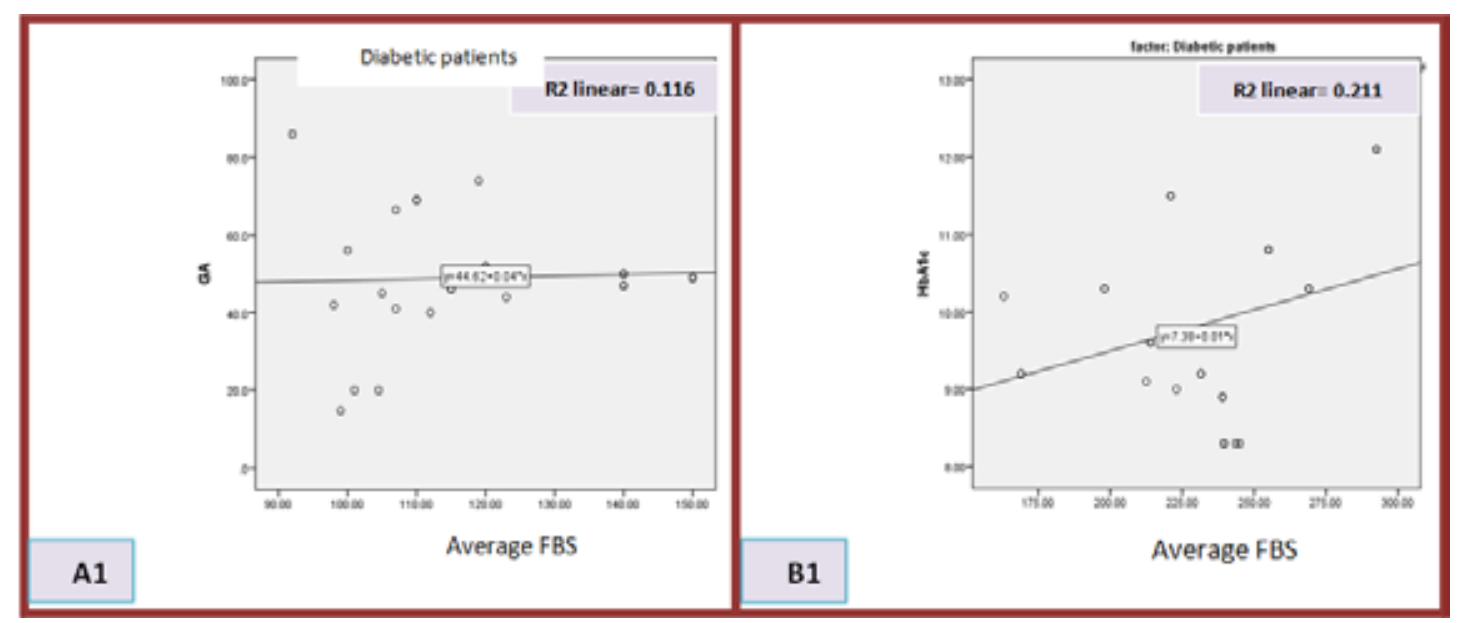

Figure (1):Correlation between average FBS values with GA\% (Figure A2) or average FBS values with $\mathrm{HbA1c \%}$ (Figure B2) in diabetic patients group. 


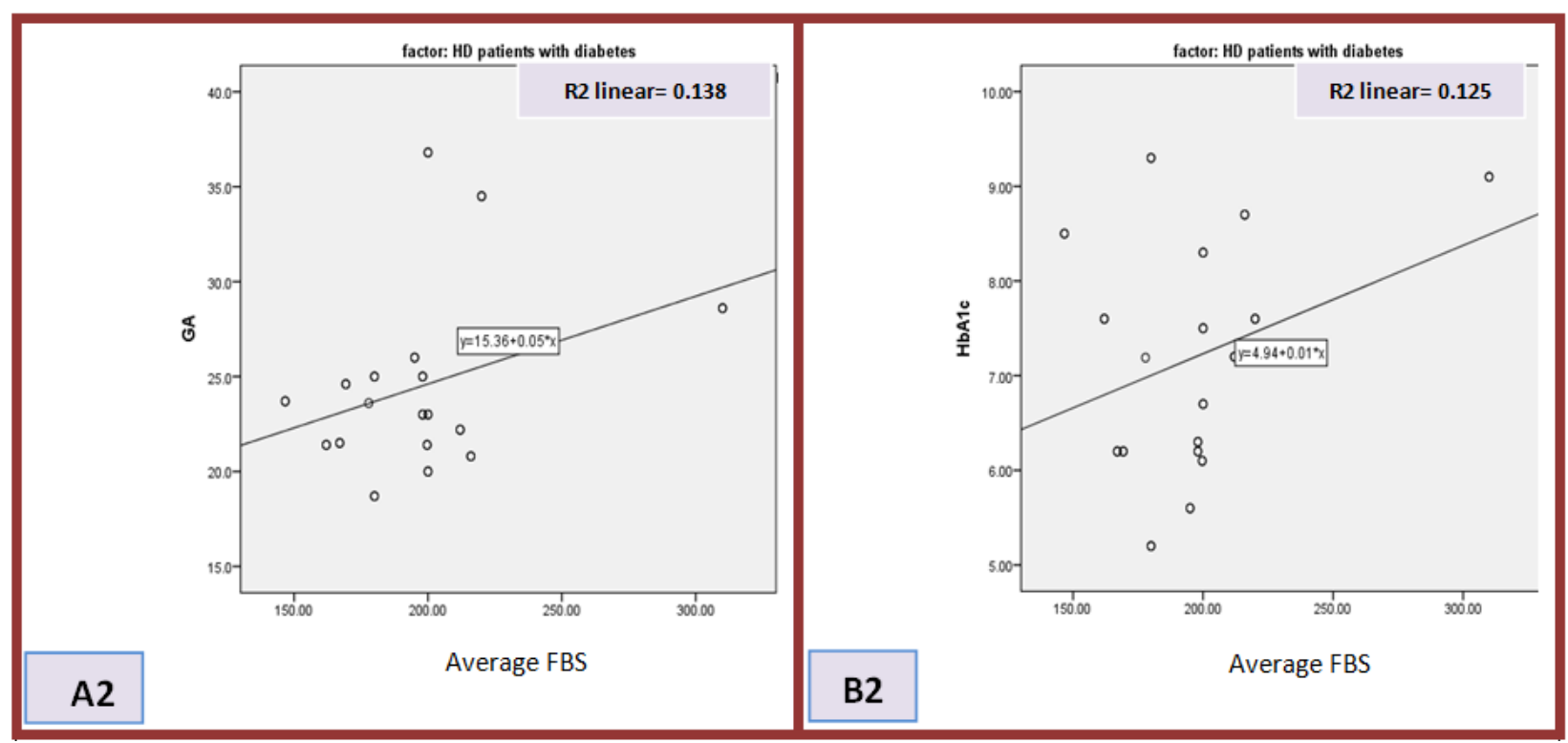

Figure (2): Correlation between the average FBS values with GA \% (Figure A3) or average FBS values with HbA1c\% (Figure B3) in HD patients with diabetic group.

Effect of a Single HD Session on GA\% and HbA1c\%: These results show that serum GA\% were almost identical between before and after a single HD session in HD patients with diabetes were $(29.4 \pm 4.71$ and $29.3 \pm 4.5$ ) respectively. While HbA1c also identical between before and after a single HD but to a lesser degree from GA, where in HD patients with diabetes were $(8.5 \pm 1.83$ and $8.19 \pm 1.2)$ respectively.

The ratio of GA/HbA1C in study groups: The GA/ $\mathrm{HbA1c}$ ratio was (3.0) in control groups and (2.93) in diabetic patients with no significant difference between these two groups. Also the GA/HbA1C ratio showed no significant difference between HD patients with diabetes (3.5) and HD patients without diabetes (3.3).

The results in figures $(1,2)$ identify with the finding of study by Inaba et al., ${ }^{(8)}$ who recorded that the significant and positive correlations between average FBS with serum GA. Also identify with Gan et al., ${ }^{(4)}$ who found that GA showed a strong correlation with mean FBS and supply a more reliable marker of glycemic control, compared with HbA1c which showed heterogeneity correlation with average FBS was indicated in HD patients. The results in table (5) agreed with Gan et al., ${ }^{(4)}$ who indicated that the GA is superior to $\mathrm{HbA} 1 \mathrm{C}$ in estimating glycemic control in HD patients with diabetes. While $\mathrm{GA} / \mathrm{HbA}$ 1c ratio symmetrical with Inaba et al., ${ }^{(8)}$ who recorded that $\mathrm{GA} / \mathrm{HbA}$ lc ratio was (2.9) in diabetic patients group and it raised significantly in the HD patients with diabetes was (3.81).
These data clearly indicated that the substances that accumulated into uremic serum did not affect GA values at all. The only factor that associated independently with GA value was the average FBS, which associated to a greater degree with GA compared with HbA1c ${ }^{(16)}$. Although the specificity and positive predictive value of HbA1c were acceptable, they were not promising; thus, to confirm diagnosis, we can not only rely on a slight elevation of $\mathrm{HbA} 1 \mathrm{c}$. The elevated $\mathrm{GA} / \mathrm{HbA} 1 \mathrm{c}$ ratio in diabetic dialysis patients, relative to diabetic subjects without nephropathy, strongly suggests that $\mathrm{HbA1c}$ was falsely reduced in diabetic subjects on hemodialysis. HD patients draw iron and vitamin B12 for the purpose of anemia correction such as external ESA. These treatment increases the development of immature RBC, which ultimately changes the original structure of the hemoglobin. Such RBCs are given inadequate exposure time to go through the process of glycosylation, which may result in lower concentration of HbAlc. The lower level of $\mathrm{HbA} 1 \mathrm{c}$ may thus offer a false impression about the overall glycemic regulation ${ }^{(15 ; 16) \text {. }}$

Conclusion: GA is a better marker for detecting short term glycemic control in HD patients with diabetes and the assessment of glycemic control by $\mathrm{HbAlc}$ in those patients might lead to underestimation, where the principle of treatment of DN is based on tight control of hyperglycemia.

Ethical Clearance: In this study all patients provided written informed consent before participation 
in this study, which was approved by institutional ethics committees (university of Anbar $\backslash$ ethical approval committee).

Source of Funding: Self.

\section{Conflict of Interest: Nil}

\section{References}

1. Freitas PA, Ehlert LR, Camargo JL. Glycated albumin: a potential biomarker in diabetes. Archives of endocrinology and metabolism. 2017 Jun;61(3):296-304.

2. George SV, Pullockara JK, Kumar SS, Mukkadan JK. A study to assess changes in the hematological profile in chronic kidney disease. The Pharma Innovation. 2015 Aug 1;4(6, Part A):1.

3. Lu CL, Ma WY, Lin YF, Shyu JF, Wang YH, Liu YM, Wu CC, Lu KC. Glycated albumin predicts long-term survival in patients undergoing hemodialysis. International journal of medical sciences. 2016;13(5):395.

4. Gan T, Liu X, Xu G. Glycated albumin versus $\mathrm{HbAlc}$ in the evaluation of glycemic control in patients with diabetes and CKD. Kidney International Reports. 2018 May 1;3(3):542-54.

5. Kim IY, Kim MJ, Lee DW, Lee SB, Rhee H, Song SH, Seong EY, Kwak IS. Glycated albumin is a more accurate glycaemic indicator than haemoglobin A1c in diabetic patients with predialysis chronic kidney disease. Nephrology. 2015 Oct;20(10):715-20.

6. Nuttall FQ. Diabetes Care. 1998; 21:1475-1480.

7. Young DS. Effects of drugs on clinical laboratory tests, $5^{\text {th }}$ ed AACC press. 2005.

8. Inaba M, Okuno S, Kumeda Y, Yamada S, Imanishi Y, Tabata T, Okamura M, Okada S, Yamakawa T, Ishimura E, Nishizawa Y. Glycated albumin is a better glycemic indicator than glycated hemoglobin values in hemodialysis patients with diabetes: effect of anemia and erythropoietin injection. Journal of the American Society of Nephrology. 2007 Mar 1;18(3):896-903.

9. Lin YC, Lin YC, Chen HH, Chen TW, Hsu CC, Wu MS. Determinant effects of average fasting plasma glucose on mortality in diabetic end-stage renal disease patients on maintenance hemodialysis. Kidney international reports. 2017 Jan 1;2(1):1826.

10. Khalaf DT. Evaluation of Heat Shock Protein 60 and its autoantibodies in Patients with Diabetes Mellitus Type1. M.Sc. Thesis. College of Education for Pure Sciences. University of Anbar. Iraq.2018.

11. Abid Hammed SM. Study of Relationship between Several Interleukins and Some Biochemical Parameters in Type II Diabetics Patients. Ph. Thesis. College of Science, University of Anbar.2019

12. Abdul Hadi FS, Sultan AS. The effects of type 2 diabetes mellitus on the levels of glycated hemoglobin, testosterone, leptin and calcium in Iraqi male patients. GJBB. 2016;5(2):164-70.

13. Moulani MR, Gawali PS. Effect of Iron deficiency on glycation of hemoglobin in non diabetics. Annals of Clinical Chemistry and Laboratory Medicine. 2016;2(2):4-7.

14. Chen $\mathrm{CW}$, Drechsler $\mathrm{C}$, Suntharalingam $\mathrm{P}$, Karumanchi SA, Wanner C, Berg AH. High glycated albumin and mortality in persons with diabetes mellitus on hemodialysis. Clinical chemistry. 2017 Feb 1;63(2):477-85.

15. Fukami K, Shibata R, Nakayama H, Yamada K, Okuda S, Koga M. Serum albumin-adjusted glycated albumin is a better indicator of glycaemic control in diabetic patients with end-stage renal disease not on haemodialysis. Annals of Clinical Biochemistry. 2015 Jul;52(4):488-96.

16. Zaman SB, Hossain N, Rahman AE, Islam SM. Can glycated hemoglobin act as a reliable glycemic indicator in patients with diabetic chronic kidney disease? evidence from the Northeast of Thailand. Medical Journal of Indonesia. 2017 Aug 18;26(2):102-8. 